\title{
Quantum Criticality and Incipient Phase Separation in the Thermodynamic Properties of the Hubbard Model
}

\author{
By D. Galanakis ${ }^{1}$, E. Khatami ${ }^{2}$, K. Mikelsons ${ }^{2}$, A. Macridin ${ }^{3}$, J. \\ MORENO $^{1}$, D. A. BROWNE ${ }^{1}$ AND M. JARRELL ${ }^{1}$ \\ ${ }^{1}$ Department of Physics and Astronomy, Louisiana State University, Baton \\ Rouge, Louisiana, 70803, USA \\ ${ }^{2}$ Department of Physics, Georgetown University, Washington, District of \\ Columbia, 2005\%, USA \\ ${ }^{3}$ Fermilab, P. O. Box 500, Batavia, Illinois, 60510, USA
}

Transport measurements on the cuprates suggest the presence of a quantum critical point hiding underneath the superconducting dome near optimal hole doping. We provide numerical evidence in support of this scenario via a dynamical cluster quantum Monte Carlo study of the extended two-dimensional Hubbard model. Single particle quantities, such as the spectral function, the quasiparticle weight and the entropy, display a crossover between two distinct ground states: a Fermi liquid at low filling and a non-Fermi liquid with a pseudogap at high filling. Both states are found to cross over to a marginal Fermi-liquid state at higher temperatures. For finite next-nearest-neighbor hopping $t^{\prime}$ we find a classical critical point at temperature $T_{c}$. This classical critical point is found to be associated with a phase separation transition between a compressible Mott gas and an incompressible Mott liquid corresponding to the Fermi liquid and the pseudogap state, respectively. Since the critical temperature $T_{c}$ extrapolates to zero as $t^{\prime}$ vanishes, we conclude that a quantum critical point connects the Fermi-liquid to the pseudogap region, and that the marginal-Fermi-liquid behavior in its vicinity is the analogous of the supercritical region in the liquid-gas transition.

Keywords: Quantum criticality, DCA, Cluster methods

\section{Introduction}

(a) Relevance of quantum criticality in the cuprates

The unusually high superconducting transition temperature of the hole doped cuprates (Bednorz \& Müller, 1986) remains an unsolved puzzle, despite more than two decades of intense theoretical and experimental research. Pairing, which has a $d$-wave symmetry and short coherence length, but too high of a $T_{c}$ to be accounted by BCS (Bardeen et al., 1957), is not the only unconventional property of these materials. Their phase diagram, shown in Fig. 1, is a landscape of exotic states of matter. Undoped cuprates are Mott insulators with antiferromagnetic long-range order (Néel, 1949). Antiferromagnetism collapses upon small doping and it is replaced by a pseudogap state characterized by a suppression of spectral weight along 
the antinodal direction. Further doping turns the system into a conventional Fermiliquid metal. Between the Fermi-liquid and the pseudogap region lies a strange metal phase with linear- $T$ resistivity. The superconducting dome emerges in the cross-over between the pseudogap and the Fermi-liquid regions at lower temperatures.

Strong electronic correlations are the cause of the rich phase diagram of cuprate superconductors (Phillips, 2010). The same strong correlations render traditional theoretical approaches, such as perturbation theory and Fermi-liquid theory, inapplicable. Some recent conceptual progress has been achieved by associating the optimal $T_{c}$ with a quantum critical point (QCP), lying underneath the superconducting dome and connecting the pseudogap and the Fermi-liquid regions (Broun, 2008; Sachdev, 2010). Unlike a classical critical point, a QCP affects the behavior of the system in a wide range of temperatures and might explain the emergence of a linear- $T$ resistivity up to room temperature.

Experimental evidence for a QCP comes from transport (van der Marel et al., 2003; Daou et al., 2009; (Balakirev et al., 2009) and thermodynamic measurements (Bernhard et al., 2001). Angle-resolved photo-emission spectroscopy (ARPES) (Shen et al., 2005; Platé et al., 2005) and quantum oscillation measurements (Doiron-Leyraud et al., 2007) show that in the pseudogap region, the Fermi surface consists of small pockets which have different topology than the large Fermi surface present in the Fermi liquid. It is reasonable to assume that those two states are orthogonal to one another and are connected through a transition. Additional evidence in support of quantum criticality comes from measurements of the Kerr signal in YBCO by Jing Xia et al. (Xia et al., 2008). They find that at the pseudogap crossover temperature, $T^{*}$ a non-zero Kerr signal develops sharply and persists even inside the superconducting dome. This is consistent with earlier neutron scattering measurements by Fauqué et al.(Fauqué et al., 2006), which show the development of magnetic order in the pseudogap phase.

In this manuscript we review numerical evidence of quantum criticality in the Hubbard model, the de-facto model for the cuprates, that appeared in earlier publications. In those cited works, the Hubbard model is solved using the dynamical cluster approximation (DCA) in conjunction with several quantum Monte Carlo (QMC) cluster solvers. In all calculations relevant for the phase diagram we neglect the superconducting transition. The interplay between the QCP and superconductivity will be discussed in a future publication. (Yang et al., 2010) In this review we focus on the thermodynamic quantities, such as the entropy and the chemical potential, and also on single-particle quantities, such as the spectral weight and the quasiparticle weight. The thermodynamic properties give unbiased evidence of quantum criticality, whereas single-particle properties may be used to gain more detailed insight on the ground state. Both set of quantities rely on the evaluation of the self-energy which can be calculated using quantum cluster methods.

At a critical interaction-dependent filling, we find that the entropy exhibits a maximum, the quasiparticle weight displays a crossover from Fermi liquid to pseudogap behavior, and the spectral function shows a wide saddle point region crossing the chemical potential. This is consistent with the presence of a QCP, since the lack of an energy scale results in an enhanced entropy at low temperatures. We also find that by tuning an appropriate control parameter, the next-nearestneighbor hoping, $t^{\prime}$, the QCP becomes a classical critical point associated with a phase separation transition. We present our findings in two sections. In section 2 , 


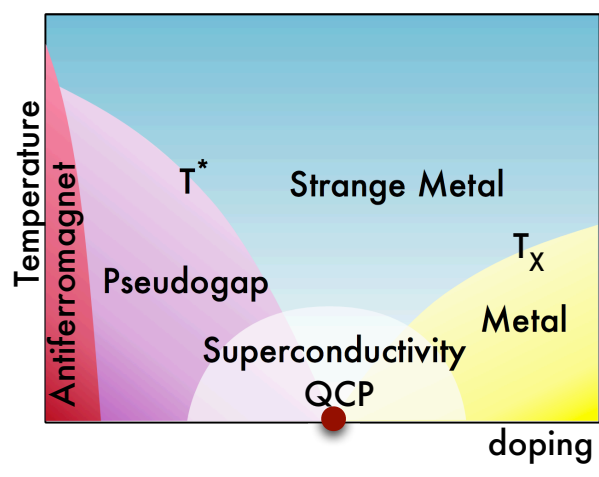

we discuss the single-particle spectra and the thermodynamics properties of the $t^{\prime}=0$ Hubbard model. In section 3 , we discuss the phase separation in the $t^{\prime}>0$ Hubbard model.

(b) Hubbard Model

Short after the discovery of high- $T_{c}$ superconductors, Anderson Anderson, 1987) suggested that the Hubbard model captures the basic properties of the high temperature superconductors and Zhang and Rice (Zhang \& Rice, 1988) demonstrated that only a single band is needed. The single-band Hubbard model is represented by the Hamiltonian:

$$
H=-t \sum_{\langle i, j\rangle, \sigma}\left[c_{i \sigma}^{\dagger} c_{j \sigma}+\text { H.c. }\right]+U \sum_{i} n_{i \downarrow} n_{i \uparrow},
$$

where $c_{i \sigma}^{\dagger}\left(c_{i \sigma}\right)$ is the creation (annihilation) operator of an electron at site $i$ and spin $\sigma, n_{i \sigma}$ is the corresponding number operator, $t$ is the hopping parameter between nearest-neighbor sites, and $U$ the on-site Coulomb repulsion. Despite its apparent simplicity, the Hubbard model is notoriously difficult to solve. No analytical solutions exist except in one dimension (Lieb \& Wu, 1968; Frahm \& Korepin, 1990; Kawakami \& Yang, 1990). However, tremendous theoretical and computational efforts have resulted in approximation schemes that provide access to the physics of this model in higher dimensions. In this manuscript we also discuss results for the generalized Hubbard model which includes hopping between next-nearest neighbor with amplitude $t^{\prime}$ :

$$
H=-t \sum_{\langle i, j\rangle, \sigma}\left[c_{i \sigma}^{\dagger} c_{j \sigma}+\text { H.c. }\right]-t^{\prime} \sum_{\langle\langle i, l\rangle\rangle}\left[c_{i \sigma}^{\dagger} c_{l \sigma}+\text { H.c. }\right]+U \sum_{i} n_{i \downarrow} n_{i \uparrow} .
$$

Important progress in our understanding of strongly correlated models has been achieved by the development of finite size methods, including exact diagonalization and QMC. The latter works well in the simulation of bosonic systems where creation and annihilation operators commute. However, due to the minus sign problem associated with the anticommutation relations of fermionic operators, QMC is limited to small lattice sizes and consequently give questionable predictions for correlated electronic systems in the thermodynamic limit. 
Another successful approach is the dynamical mean-field approximation (DMFA) which treats the local dynamical correlations explicitly and non-local (inter-site) correlations in a mean-field approximation (Georges et al., 1996; Metzner \& Vollhardt, 1989; Müller-Hartmann, $1989 a, b$ ). This technique becomes exact in the limit of infinite dimensions (Georges \& Kotliar, 1992; Jarrell, 1992). However, when applied to finite dimensions, the DMFA fails to describe the renormalization effects due to momentum-dependent modes and the transitions to phases with non-local order parameters. Thus, DMFA misses physical phenomena that are abundant in strongly correlated systems, such as the development of spin or charge density wave phases, localization in the presence of disorder, spin-liquid physics, unconventional superconductivity, etc.

The limitations of the DMFA are addressed by cluster mean-field theories. Those fall into two categories (Maier et al., 2005): the cluster dynamical mean field theory (CDMFT) (Kotliar et al., 2001), which is formulated in real space, and the DCA (Hettler et al., 1998) which is formulated in momentum space. In both cases the system is viewed as a cluster embedded in an effective medium. The formal difference between DCA and CDMFT is that in real space, the DCA cluster satisfies periodic boundary conditions whereas the CDMFT cluster is open. The two methods should give the same results for large enough clusters. In this work we present DCA (Hettler et al., 1998, 2000) results.

DCA treats short-ranged correlations explicitly, while longer ranged ones are approximated by the mean field. By increasing the cluster size, the length-scale of the explicitly treated correlations can be gradually increased while the calculation remains in the thermodynamic limit. In momentum space, the DCA can easily be conceptualized as the approximation in which the self-energy calculated by the coarse grained green function. Quantum Monte Carlo based solvers such as HirschFye (HFQMC) (Hirsch \& Fye, 1986), continuous-time (CTQMC) (Rubtsov et al., 2005) and determinantal quantum Monte Carlo (DQMC) (Blankenbecler et al., 1981) are used to solve the cluster problem. QMC methods are often formulated in imaginary time and an analytic continuation to real time is necessary to evaluate physical quantities. Fortunately, powerful techniques such as the maximum entropy method (MEM) (Gubernatis et al., 1991; Jarrell \& Gubernatis, 1996) are able to successfully select the most likely solution.

Even though quantum cluster schemes have provided a tremendous breakthrough in our understanding of the Hubbard model, they are also subject to limitations. Quantum Monte Carlo solvers suffer from the sign problem, which scales exponentially with inverse temperature, interaction strength and cluster size. This limits the application of the method to relatively small cluster sizes, higher temperatures and intermediate interactions. The limitation in the cluster size is particularly problematic close to a phase transition where the correlation length diverges. The coarsegraining also limits the momentum resolution, which for typical cluster sizes is too small to capture detail features of the spectra, such as van Hove singularities. For a Fermi liquid, this is not a limitation since the physics is dominated by the low frequencies in which the self-energy is momentum independent. However, intrinsically anisotropic states, such as the pseudogap, or possibly the quantum critical region, can be captured only approximately. Finally, MEM uses Bayesian statistics to find the most likely spectra for the QMC data, subject to sum rules, such as 
conservation of the spectral weight. Because of the statistical errors in the QMC data, the frequency spectrum resolved using MEM has a limited resolution.

Despite those limitations, progress can be achieved in accessing the quantum critical region by algorithmic optimizations. A truly universal way in dealing with the severity of the sign problem is to vastly increase the statistics, using massively parallel QMC algorithms with highly optimized codes.

\section{From Fermi Liquid to Pseudogap}

A great advantage of the DCA is its ability to evaluate the self-energy as a function of momentum $\mathbf{k}$ and Matsubara frequency $i \omega_{n}, \Sigma\left(\mathbf{k}, i \omega_{n}\right)$. From the self-energy various single-particle quantities, such as the spectral function, $A(\mathbf{k}, \omega)$, the quasiparticle weight, $Z_{\mathbf{k}}$, and the energy can be derived. All those quantities provide insight about the ground state of the system. In this section we will show how the transition from the Fermi-liquid to the pseudogap state is reflected in such single-particle quantities.

(a) Spectral Function

The single-particle spectral function shows a clear evolution from a Fermi-liquid to a pseudogap state as the filling increases towards half filling. Fig. 2 displays a density plot of the spectral function, $A(\mathbf{k}, \omega)=-\frac{1}{\pi} \Im G(\mathbf{k}, \omega)$, which is extracted by analytically continuing the imaginary time Green function. At low filling, $n<0.85$, the spectral function exhibits a typical Fermi-liquid form. A notable characteristic is the presence of a wide saddle point region, reminiscent of a van Hove singularity, (Radtke \& Norman, 1994) along the antinodal direction. Around the critical filling of $n=0.85$ this saddle point feature crosses the chemical potential. This crossing results in a sharp peak in the density of states (Vidhvadhiraia et al., 2009), which displays low-energy particle-hole symmetry (Chakraborty et al., 2008). We are currently exploring the influence of the van Hove singularity on the superconducting transition. (Pathak et al.) At higher filling, $n>0.85$, the spectral weight collapses along the antinodal direction and a pseudogap opens. The Fermi surface obtained by extremizing $\left|\nabla n_{\mathbf{k}}\right|$ shows a similar evolution (see lower panels in Fig. (2). The Fermi-liquid region consists of a large hole pocket, which extends and touches the edges of the Brillouin zone $(0, \pm \pi),( \pm \pi, 0)$ at $n=0.85$. In the pseudogap region the Fermi surface consists of four Fermi arcs centered around the nodal points, similar to the ones obtained from ARPES. These results clearly demonstrate that the DCA can capture qualitatively the evolution of the ground state from a Fermi-liquid to a pseudogap phase.

\section{(b) Quasiparticle Weight}

Whereas the spectral function gives a qualitative understanding of the ground state, it relies on the analytic continuation of numerical data. Since extracting quantitative information from analytically continued data is difficult, a more robust way is to rely on imaginary time quantities, such as the quasiparticle weight $Z(\mathbf{k})$. Since the quasiparticle weight is finite across a Fermi surface, but vanishes if the spectrum is incoherent, it will allows to clearly distinguish between a Fermi liquid and a pseudogap state. The quasiparticle weight can be directly obtained from the Matsubara frequency self-energy as $Z_{0}(\mathbf{k})=\left(1-\frac{\Im \Sigma\left(\mathbf{k}, i \omega_{0}\right)}{\omega_{0}}\right)^{-1}$, where $\omega_{0}=\pi T$ 

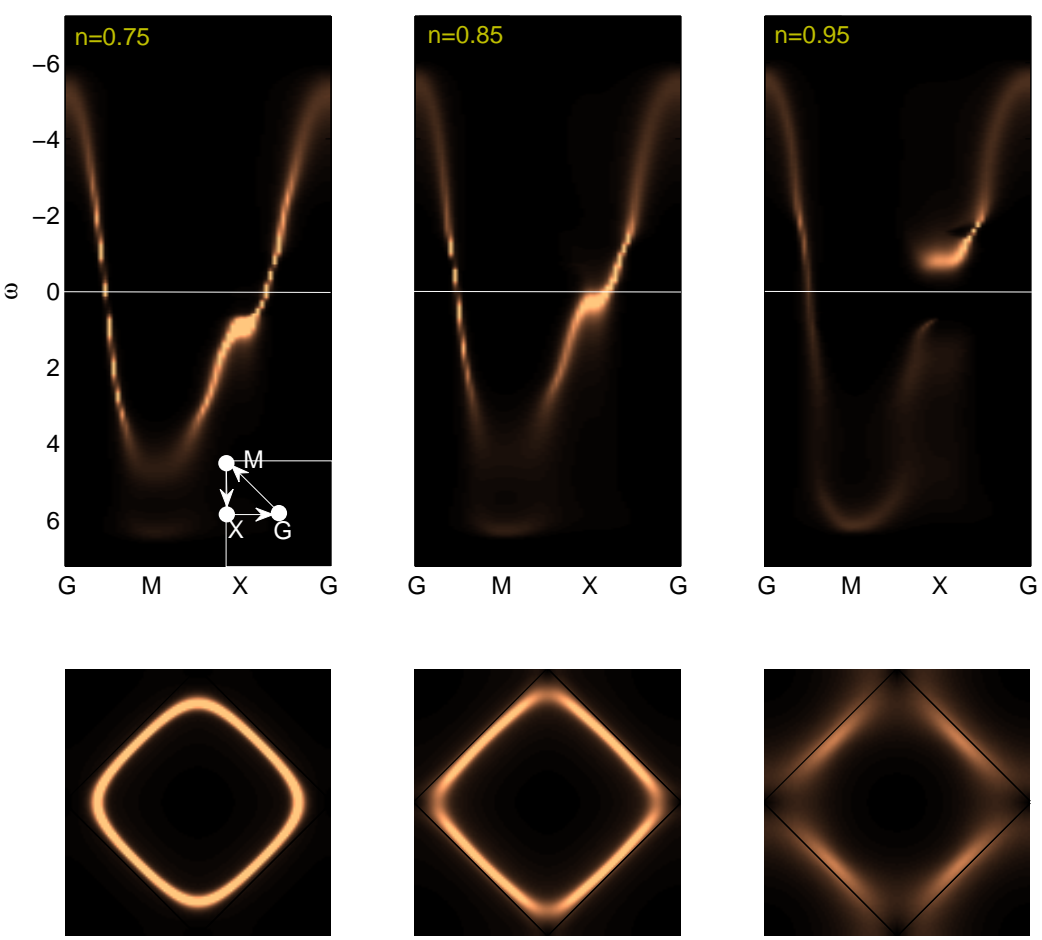

Figure 2. Upper panels: Density plots of the spectral function $A(\mathbf{k}, \omega)$ for the Fermi liquid (left), marginal Fermi liquid (middle) and pseudogap region (right) for filling $n=0.75,0.85$ and 0.95 , respectively. (The dashed feature seen in the regions of steepest dispersion, especially for $n=0.75$, is a plotting artifact). The momentum is along the path $G(0,0) \rightarrow M(\pi, \pi) \rightarrow X(\pi, 0) \rightarrow G(0,0)$. A wide saddle point region between X and $\mathrm{G}$ sits above the chemical potential in the Fermi-liquid region and crosses it around the critical filling $(n=0.85)$. In the pseudogap region this features sits below the chemical potential leaving a gap along the antinodal direction behind it. Note that the fact that the dispersion looks discontinuous along $G(0,0) \rightarrow M(\pi, \pi)$ in the left and middle panels is an artifact of our interpolation algorithm. Lower panels: Fermi surface as extracted from $\left|\nabla n_{\mathbf{k}}\right|$ in the Fermi liquid (left), marginal Fermi liquid (middle) and pseudogap (right) region showing the development of the pseudogap in the antinodal direction. The Coulomb repulsion is $U=6 t$, the temperature $T=0.069 t$, and the cluster size $N_{c}=16$. The energy unit is $4 t$.

is the lowest fermionic Matsubara frequency. At the limit $T \rightarrow 0$ and for a wellbehaved self-energy, $Z_{0}(\mathbf{k})$ converges to the quasiparticle weight, $Z(\mathbf{k})$. Fig. 3 (a) displays $Z_{A N}=Z_{0}\left(\omega_{0}=\pi T, k \|(0,0) \rightarrow(0, \pi)\right)$, the Matsubara quasiparticle weight along the antinodal momentum direction for $U=6 t$ and a cluster of size $N_{c}=16$ (Vidhyadhiraia et al., 2009). The momentum $\mathbf{k}$ at the Fermi surface is determined by maximizing $|\nabla n(\mathbf{k})| . Z_{A N}$ exhibits two distinguishable behaviors: for $n>n_{c}=0.85$ the quasiparticle weight vanishes, whereas it approaches a finite value for $n<n_{c}$. The $n>n_{c}$ region corresponds to the pseudogap state in which 

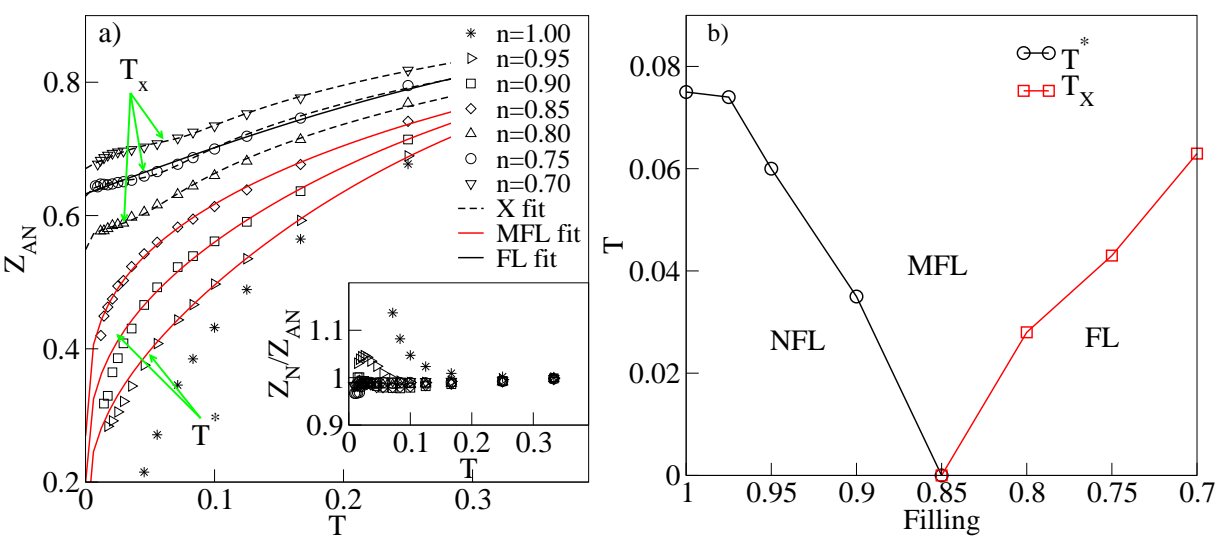

Figure 3. a) The antinodal quasiparticle fraction $Z_{A N}$ as a function of temperature for different values of filling, $U=6 t$ and cluster size $N_{c}=16$ (the unit of energy is $4 t$ ). The onset of the pseudogap region is determined by the vanishing of the antinodal spectral weight at zero temperature. The dashed and solid lines represent fits of the low temperature $(T<0.3)$ data to marginal Fermi liquid (red solid curves), Fermi liquid (black solid curves) and crossover forms (dashed black curves), respectively. The arrows show the corresponding crossover temperatures $T_{X}$ and $T^{*}$. The value of $T^{*}$ presented here is obtained from the spin susceptibility as explained in (Vidhvadhiraia et al., 2009), but is consistent with the one extracted from the from the fitting forms. The ratio $Z_{N} / Z_{A N}$ of the quasiparticle weight in the nodal $((\pi, \pi))$ and antinodal $((0, \pi))$ directions (inset) diverges as the pseudogap develops in accordance with Fig. 2. b) The crossover temperatures $T_{X}$ and $T^{*}$ as a function of filling as extracted from the temperature dependence of $Z_{A N}$ (Vidhvadhiraia et al., 2009) for the same parameters.

the spectral weight collapses along the antinodal direction, while the $n<n_{c}$ region behaves as a Fermi liquid.

The temperature dependence of $Z_{A N}$ (Fig. 3 (a)) not only provides information about the ground state but also allows the extraction of relevant energy scales. By comparing the numerical results with analytical expressions derived from particular phenomenological forms of the self-energy, we obtain $T_{X}$ and $T^{*}$. At low filling, $n<n_{c}$, the high $T$ dependence of $Z_{A N}$ is best fit by a marginal Fermi-liquid form, whereas for low $T$ the data is best fit by a Fermi liquid. The crossover occurs at a temperature $T_{X}$, which is extracted by fitting with a crossover function, and is accompanied by a change in the sign of the curvature of $Z_{A N}$. At higher filling $(n>0.85)$, the high temperature $Z_{A N}$ can also be fit by a marginal Fermi liquid, whereas at low temperatures, it cannot. The crossover temperature $T^{*}$ can be extracted as the lowest temperature where the marginal Fermi liquid fit lies within the statistical error. However a more accurate value can be obtained from the bulk spin susceptibility which exhibits a peak at $T^{*}$ and the two values are found to be consistent (Vidhyadhiraja et al., 2009). The crossover temperatures $T_{X}$ and $T^{*}$ are shown in Fig. 3 (b). Both of them converge to zero as the filling approaches $n_{c}=0.85$, which is the same value for which the peak in the density of states (Vidhyadhiraja et al., 2009) crosses the chemical potential.

(c) Thermodynamics

A different perspective at the transition from a Fermi liquid to the pseudogap 


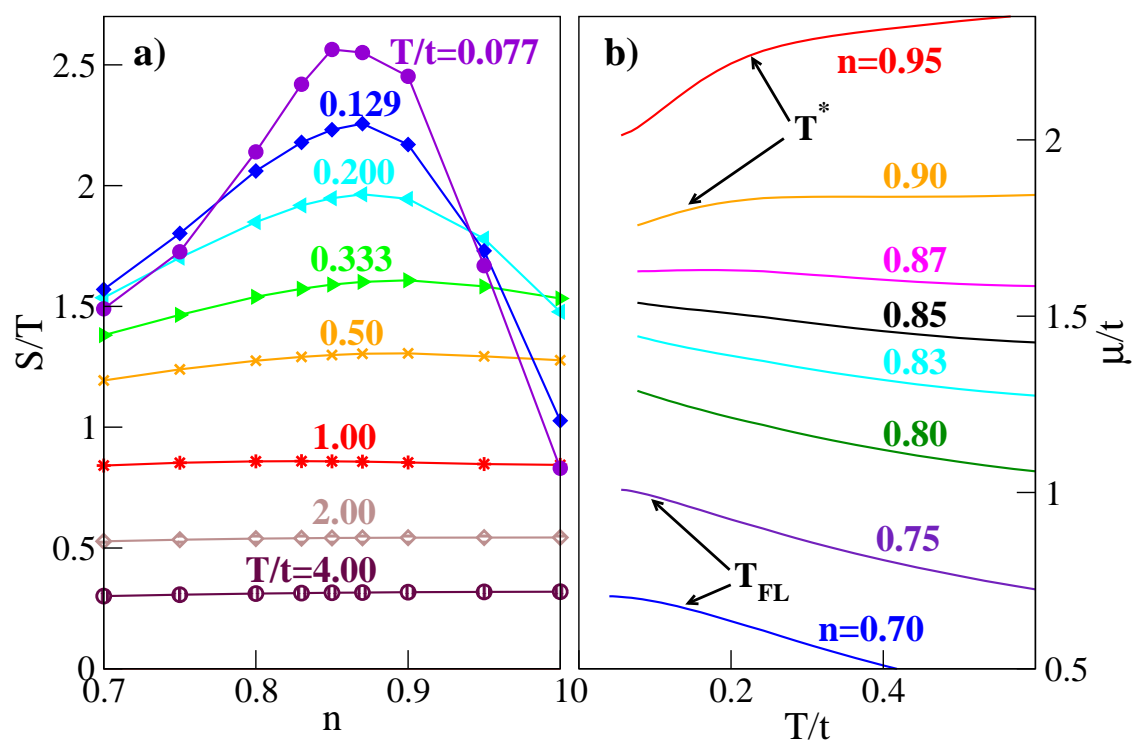

Figure 4. a) The filling dependence of the entropy divided by temperature, $S / T$, for various temperatures at $U=6 t$ and $N_{c}=16$. With decreasing temperature a peak develops around the critical filling of $n_{c}=0.85$. b) The temperature dependence of the chemical potential $\mu$ for different fillings. At the critical filling, $n_{c}, \mu$ becomes temperature independent at low temperatures.

state comes from the evaluation of the entropy. We obtain the entropy by integrating the energy using the formula:

$$
S(\beta, n)=S(0, n)+\beta E(\beta, n)-\int_{0}^{\beta} E\left(\beta^{\prime}, n\right) d \beta^{\prime},
$$

where $\beta$ is inverse temperature and $S(0, n)$ is the infinite temperature entropy. Equation 2.1 is appropriate for QMC calculations, because the integration reduces the statistical error. The challenge is to have good enough statistics to control the error of the surface term, $\beta E(\beta, n)$. In Mikelsons et al. (Mikelsons et al., 2009) large statistics was possible simply by using large computational resources. The entropy divided by the temperature, shown in Fig. 团(a), exhibits a maximum at exactly the same critical filling that was identified before from the spectral function and the quasiparticle weight. In Fig. 团(b), we show the chemical potential, $\mu$, as a function of temperature. We note that at the critical filling $d \mu / d T=0$, since the entropy and the chemical potential are related by the Maxwell relation:

$$
\left(\frac{\partial S}{\partial n}\right)_{T, U}=-\left(\frac{\partial \mu}{\partial T}\right)_{U, n}
$$

Also the temperature dependence of the chemical potential can be used as a practical criterion to identify the location of the critical filling, because evaluating the chemical potential is much less computationally intensive than evaluating the entropy. Using this criterion we investigate the important question of the de-

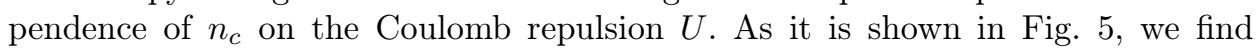



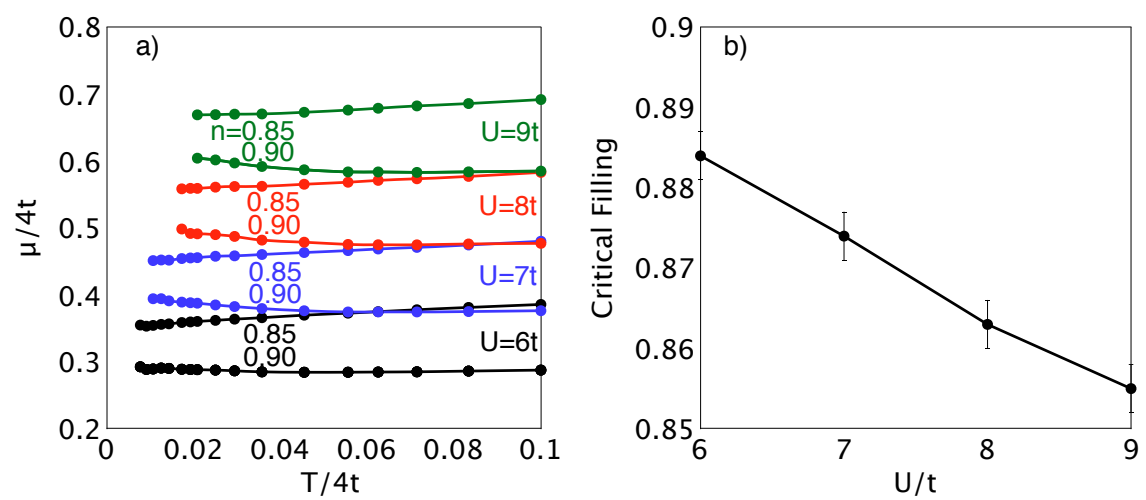

Figure 5. a) The chemical potential as a function of temperature for fillings of $n=0.85$ and 0.90 and for a variety of interaction strengths $U$ for $N_{c}=12$. b) The critical filling, defined by the filling in which $\partial \mu / \partial T=0$ versus $U$. The critical filling decreases with $U$ monotonically and is projected to reach the atomic limit value of $n_{c}=2 / 3$ at $U_{c}=30 t$.

that increasing $U$ reduces the critical filling and thus enlarges the pseudogap region in the phase diagram. Our results follow the trend proposed in earlier arguments (Chakraborty et al., 2008) according to which the critical filling decreases in order to reach the atomic limit value of $n_{c}=2 / 3$.

In this section we have shown that several single-particle quantities are consistent with the presence of a QCP. The qualitative form of the single-particle spectrum shown in Fig. 2 is fundamentally different in the Fermi-liquid and the pseudogap regions, which points to orthogonal ground states. The temperature dependence of the quasiparticle weight reveals the presence of two crossover temperatures $T^{*}$ and $T_{X}$, which converge to zero at $n_{c}$ as shown in Fig. 3 (b). If the crossover temperatures $T_{X}$ and $T^{*}$ constitute energy scales that suppress degrees of freedom, their vanishing at $n_{c}$ means that there are no relevant energy scales to quench the entropy and therefore it collapses at a slower rate, which is consistent with the peak of the entropy observed at $n_{c}$. The natural next step to investigate quantum criticality is to access the QCP. However the fermion sign problem severely limits the applicability of quantum Monte Carlo techniques close to a QCP. It is possible however, as we will discuss in the next section, that by tuning an appropriate control parameter, the critical point may be lifted to finite temperature and thus studied with QMC.

\section{Phase Separation and Quantum Criticality}

Experiments suggest that cuprate superconductors are susceptible to charge inhomogeneities, such as stripes or checkerboard modulations (Hinkov et al., 2004). These inhomogeneous charge patterns have stimulated intense theoretical and experimental research. Here we will consider the possibility that those charge instabilities are evidence that the cuprates are close to a phase separation transition, and this proximity may be related to the nature of the QCP.

Our findings suggest that the Hubbard model displays a phase diagram similar to the one for the gas-liquid transition with Mott liquid (ML) and Mott Gas (MG) regions. Fig. 6(a) shows a possible phase diagram for the Hubbard model as a 
(a) $\mathrm{n}$

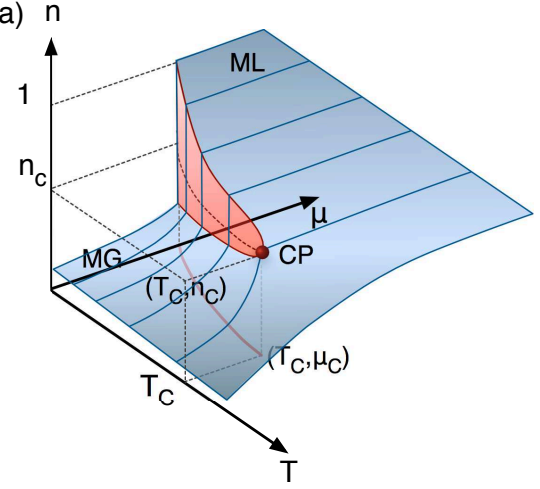

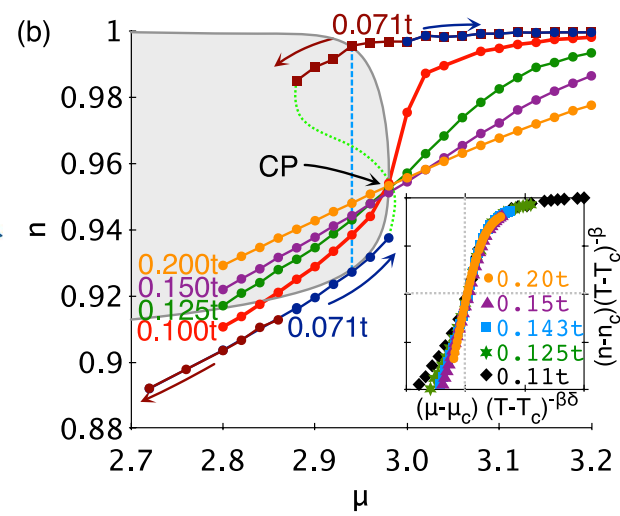

Figure 6. a) The schematic phase diagram in the presence of charge separation. This phase diagram describes the transition between two states labeled Mott Liquid (ML) and Mott Gas (MG) as a function of temperature, $T$, chemical potential, $\mu$, and filling, $n$. The red surface represents the coexistence region, which terminates in a critical point $(\mathrm{CP})$. As we go around the critical point the state changes smoothly from ML to MG. Along the first order transition line and for a fixed $T$ and $\mu$, the filling has two values. b) Filling as a function of chemical potential for several temperatures in the vicinity of the charge separation critical point. The number next to each curve represents the temperature. The coexisting phases are an incompressible Mott liquid at $n \approx 1$ and a compressible Mott gas at $n \approx 0.93$. The critical temperature is $T_{c}=0.1 t$. The blue dashed line represents the surface of metastability which is not accessible within the DCA. The green dotted line represents the isothermal of the metastable state inside the phase coexistence region (gray zone). At the critical point the isothermals for $T>T_{c}$ cross. The inset shows the scaling curve $\left(n-n_{c}\right)\left(T-T_{c}\right)^{-\beta}$ vs $\left(\mu-\mu_{c}\right)\left(T-T_{c}\right)^{-\beta \delta}$ in arbitrary units for $\mu_{c}=3 t, n_{c}=0.96$, $T_{c}=0.1 t$. The scaling exponents, $\beta=0.10 \pm 0.05$ and $\beta \delta \sim 1$, are roughly consistent with the Ising universality class.

function of $T,|\mu|$, and $n$. The red-colored surface is a schematic of the region where the Mott liquid and Mott gas states, characterized by different densities, coexist for $T<T_{c}$. The critical point is located at temperature $T_{c}$, filling $n_{c}$, and chemical potential $\mu_{c}$. One can go from one state to the other either smoothly, by avoiding the phase separation region, or through a first-order transition by crossing it. Right on the phase separation region, the density has two values for a given value of $\mu$ and $T$.

Macridin et al. (Macridin et al., 2006) provided compelling evidence of phase separation in the case of the generalized Hubbard model (Eq. (1.2)) with positive next-near-neighbor hopping $t^{\prime}=0.3 t$ and $U=8 t$. Using the DCA in a $N_{c}=8$ cluster with HFQMC as the cluster solver, they showed that below a critical temperature $T_{c} \sim 0.1 t$ a first order transition occurs, which is identified by a hysteresis in the $n$ versus $\mu$ curve for $T<T_{c}$. As shown in Fig. 6)(b) with more precise data obtained using DQMC as the cluster solver, the hysteresis is between two states of different filling, the Mott liquid at half filling and the Mott gas at a filling of about 0.93 for $T=0.071 t$. The Mott liquid is incompressible and insulating. Its compressibility, which is the slope of the filling vs $\mu$ curve in the high filling side of the hysteresis curve, is small and decreases with temperature. Also the density of states of the ML phase, shown in Fig. 7(a), exhibits a gap as expected for an 


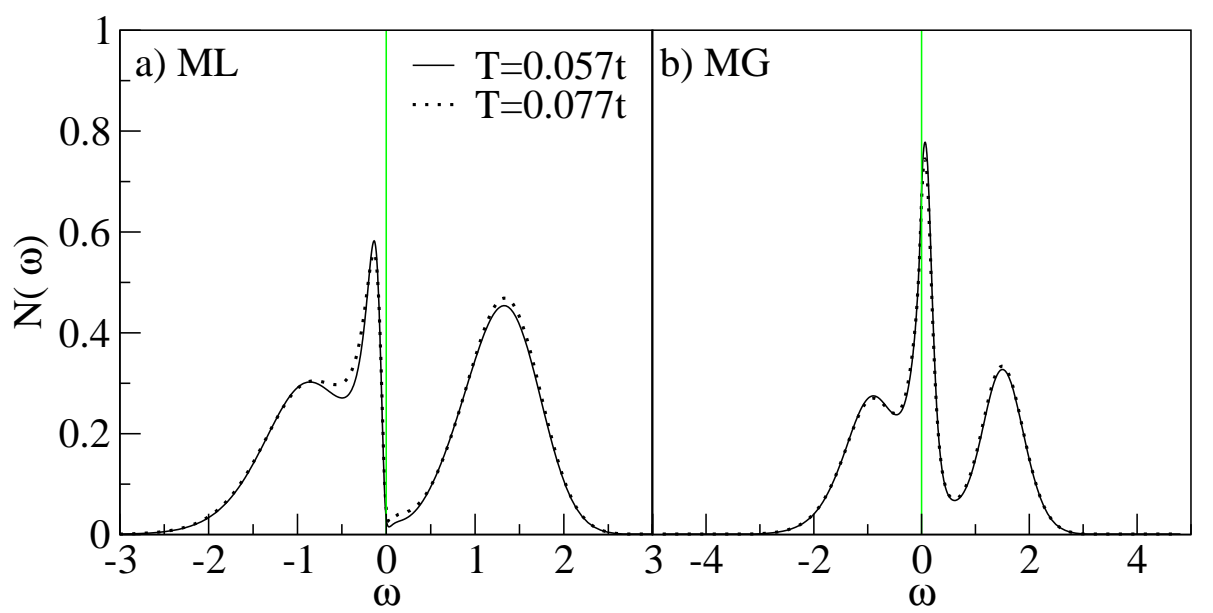

Figure 7. The density of states of the a) Mott liquid and b) Mott gas states at $T=0.077 t$ (dotted line) and $T=0.057$ (solid line). The Mott liquid is an incompressible insulator with a pseudogap while the Mott gas is weakly compressible with a Fermi liquid peak in the DOS.

insulator. On the other hand, the Mott gas is compressible and metallic; the density of states is finite at the chemical potential $(\mu=\omega=0)$, as displayed in Fig. 77(b).

The analogy to the well-known phase diagram of a liquid-gas mixture, such as water and steam, is useful to understand this phase transition. At low temperatures, there is a region in the pressure-volume phase diagram in which water and steam coexist for a range of pressures. As the temperature is increased, the region of coexistence contracts and finally terminates at a critical point where the compressibility diverges. In the pressure-temperature phase diagram, this region of coexistence becomes a line of first order transitions which terminates at a second order point where the water and gas become indistinguishable and the compressibility diverges. Since the line terminates, it is possible for the system to evolve adiabatically from steam to water without crossing a phase transition line; therefore, the steam and water must have the same symmetry.

In the Mott liquid and Mott gas system the chemical potential $\mu$ replaces the pressure and the density $n$ replaces the volume of the water-gas mixture. Because the order parameter separating the ML from the MG, the density $n$, does not have a continuous symmetry, order may occur at finite temperatures, and the MLMG transition will most likely be in the Ising or lattice gas universality class. Within this context, one may then understand the hysteresis of Fig. 6(b). The solid lines are isotherms which show how the system evolves with increasing density. At the temperature $T=T_{c}$, the compressibility diverges at the critical filling. As the temperature is lowered further, there is a region where the ML and MG coexist. Inside this region the isothermals contain unphysical regions of negative compressibility (dashed green line in Fig. 6(b)) along with metastable regions of positive compressibility. The metastable branch of the isothermal in the vicinity of the ML is a "supercooled" ML, whereas the one in the vicinity of the MG is a "superheated" MG. The translational invariance of DCA along with the stabilizing effect of the mean-field host enable access to those metastable states. However 


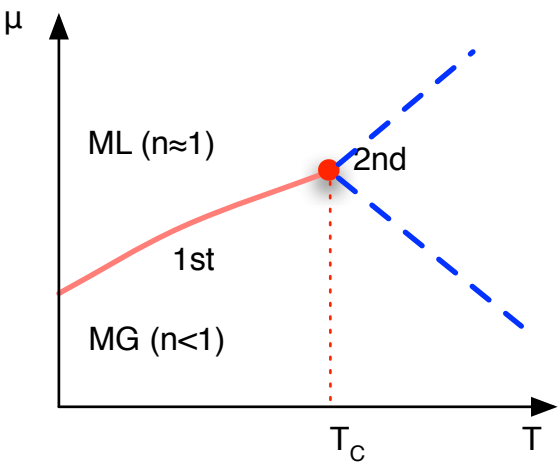

(a)

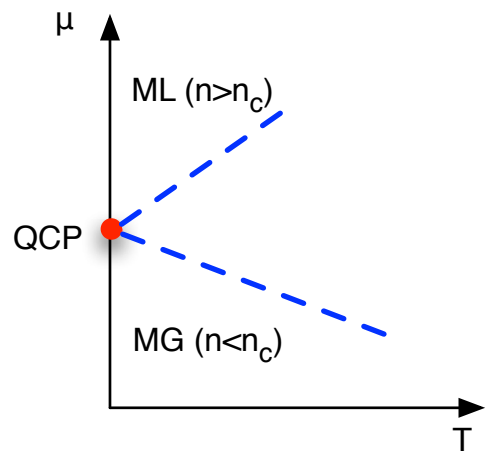

(b)

Figure 8. (a) The chemical potential-temperature phase diagram of the ML and MG mixture for $t^{\prime}>0$. The ML and MG coexist on a line of first order transitions with positive slope. Since ML and MG have the same symmetry this line can terminate in a second order critical point. The blue dashed lines define the boundaries of the supercritical region where the ML and MG cannot be distinguished. Outside this region either the ML or MG character dominates. (b) The chemical potential-temperature phase diagram for $t^{\prime} \rightarrow 0$. The first-order line is absent but supercritical region remains as a quantum critical region. In the Hubbard model the lines $T *$ and $T_{X}$ (Fig. 3 (b)) define the boundaries of this region.

the real physical system will phase separate and the two phases will co-exist in equilibrium (dotted blue line in Fig. 6(b)).

We can sketch the phase diagram in the $T-\mu$ plane using the analogy to the water-steam mixture. The most generally applicable rule governing the shape of phase diagrams was established by Gibbs. For a system of $c$ conserved components and $f$ phases, the Gibbs constraint is give by the relation $\Phi=c-f+2$ where $\Phi$ is the number of independent variables needed to specify the state of every phase. In this case, as in the water-steam system, the number of components $c=1$, since the particle number is conserved. At a location in the phase diagram where only one phase exists, $\Phi=1-1+2=2$, so there are two independent variables, and the phase diagram is a surface on the $\mu, T$ and $n$ three-dimensional space. There will be places in the phase diagram where two phases exist simultaneously, then $\Phi=1-2+2=1$, implying that two phases co-exist only along lines in the phase diagram. At the lines in the $T-\mu$ plane where two phases coexist, $n$ is also determined for each phase, but its value can be different. That is a line of first order transitions.

Additional information about the lines of first order transitions is obtained from the Clapeyron's equation. The Gibbs free energy $G=E-T S-\mu N$, and $d G=$ $-S d T-N d \mu$, must be the same for the coexisting phases on a line. If we label the two phases 1 and 2 , then

$$
\left(S_{1}-S_{2}\right) d T=-\left(N_{1}-N_{2}\right) d \mu .
$$

If we identify the latent heat $L=\left(S_{1}-S_{2}\right) T$, then $d \mu / d T=-L /(T \Delta n)$ represents the slope of the first order transition line. Since the latent heat $L$ of going from 

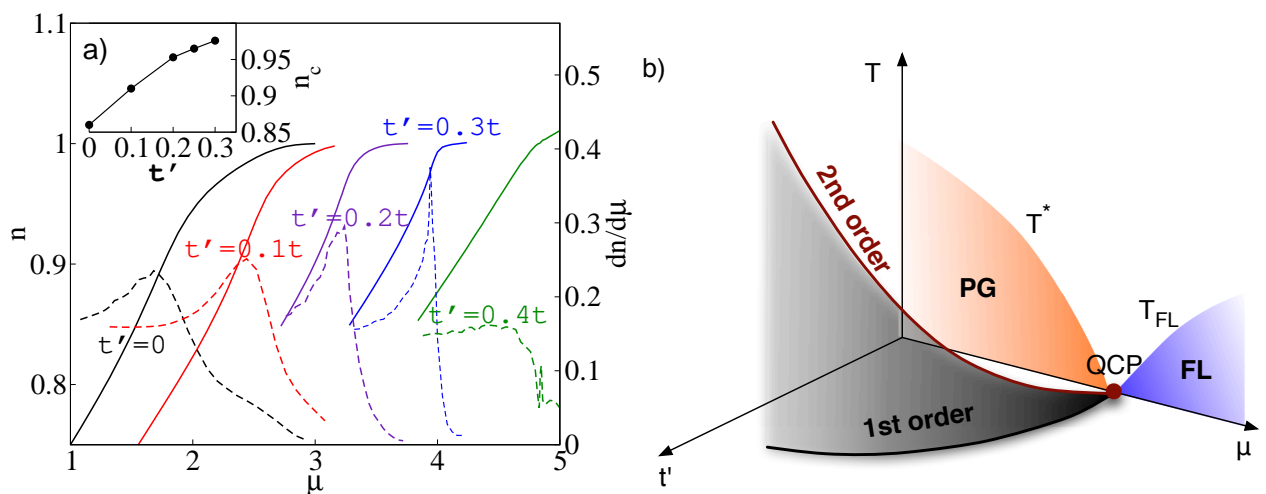

Figure 9. (a) Filling, $n$, vs. chemical potential, $\mu$, for $T=0.077 t, N_{c}=16, U=6 t$ and various $t^{\prime}$ is shown in solid lines and the compressibility $\frac{d n}{d \mu}$ in dashed lines. A critical filling, identified by the peak in the compressibility appears at higher temperatures and fillings as $t^{\prime}$ is increased. The inset shows the $t^{\prime}$ dependence of the critical filling, $n_{c}$. (b) Schematic phase diagram of the Hubbard model in the $\mu, t^{\prime}$ and $T$ space (neglecting superconductivity). The classical critical point turns asymptotically into a quantum critical point as $t^{\prime} \rightarrow 0$.

ML to MG is positive, but $d n$ is negative, the slope $d \mu / d T$ of the line of first order transitions is positive.

Above the critical point terminating the ML-MG transition, the system displays supercritical behavior in a region where the gas and the liquid cannot be distinguished thermodynamically (c.f. Fig. 8). It is possible for the system to evolve adiabatically through a counterclockwise path from deep in the MG region, through the supercritical region, into the ML region. At the lower edge of the supercritical region, the system loses the Fermi-liquid character of the MG, and at the upper edge, it begins to acquire the pseudogap character of the ML.

Let us discuss now how this phase separation, which occurs at finite temperature, is related to quantum criticality. The key parameter is the next-nearest-neighbor hoping, $t^{\prime}$. For $t^{\prime}=0$ there is no evidence for phase separation at finite $T$, but such a phase separation occurs for positive $t^{\prime}$. Khatami et al. (Khatami et al., 2010) performed a systematic analysis of the phase diagram of the extended Hubbard model as a function of $t^{\prime}$. As shown in Fig. 9 (a) the compressibility, $\chi_{c}=d n / d \mu$, exhibits a peak for all positive $t^{\prime}$ at a critical filling that depends on $t^{\prime}$. The width of the peak measures the distance from the critical temperature: the sharper the peak the closer to $T_{c}$ the employed temperature is. We see that the critical temperature increases with $t^{\prime}$ and it starts from $T_{c}=0$ at $t^{\prime}=0$. These results point to the phase diagram of Fig. 9 (b). At a positive $t^{\prime}$ a charge separation occurs at temperatures $T<T_{c}\left(t^{\prime}\right)$ and at a critical filling $n_{c}\left(t^{\prime}\right)$ between an incompressible and insulating Mott liquid and a compressible metallic Mott gas. Right at $T_{c}$, there is a terminating second-order critical point. By decreasing $t^{\prime}$ this second-order critical point is pushed down to lower temperatures. Presumably the line of second-order critical points terminates at the QCP.

Such a scenario constitutes a new path to quantum criticality as it is closely tied to charge fluctuations rather than spin fluctuations. However, numerous simulations suggest that a finite positive $t^{\prime}$ enhances antiferromagnetic correlations, 
and since phase separation is only present for $t^{\prime}>0$ it suggests that it is driven by strong spin correlations. In addition, previous simulations incorporating Holstein phonons to the Hubbard model found that phonons also enhance the phase separation instability (Macridin \& Jarrell, 2009). As $t^{\prime} / t \rightarrow 0$ (and the electron-phonon coupling vanishes), the phase separation critical point approaches zero temperature becoming a QCP. Here, the first-order behavior is absent from the phase diagram (Fig. 3(b)) leaving only the adiabatic path from the ML to the MG, which passes through the supercritical region, which is now the quantum critical (QC) region. The crossover scale $T_{X}$ and the pseudogap scale $T^{*}$ are now understood as the boundaries of the $\mathrm{QC}$ region. As we cross the line of $T^{*}$ from the $\mathrm{QC}$ region into the ML region, the characteristics of the ML become apparent, including the pseudogap in the DOS and the insulating behavior. As we cross the line of $T_{X}$ from the $\mathrm{QC}$ region into the $\mathrm{MG}$, the characteristics of the $\mathrm{MG}$ become apparent, including Fermi liquid formation.

Those calculations certainly do not elucidate the nature of the Mott liquid and Mott gas states in real materials. The long-ranged nature of the Coulomb interaction prevents true charge separated states, but the phase separation we observe may also correspond to other charge instabilities, such as stripes or checkerboard patterns. To distinguish between different charge instabilities, systematic calculations in much larger clusters are necessary which are not practical at the moment. However, whatever the type of order, those calculations provide convincing evidence for the existence of a first-order transition at low temperatures. Such a transition is similar to the liquid-gas or the ferromagnetic transition and its phase diagram would look like Fig. 6(a): a first order line of coexistence which terminates at a critical point at a critical temperature $T_{c}$ and a critical filling $n_{c}$.

\section{Conclusions}

The presence of a QCP at finite filling in the cuprate phase diagram is a topic of active theoretical and experimental research. Quantum cluster methods are able to shed some light in this phase diagram. By studying single-particle quantities for $t^{\prime}=0$, such as the spectral function and the entropy, it can be shown that a Fermi-liquid region at low filling and the pseudogap region at higher filling have different spectral signatures, and are connected through an intermediate "marginal Fermi-liquid" region of maximal entropy. Due to limitations of quantum Monte Carlo, the ground state and quantum criticality are not accessible. We also neglect the superconducting phase transition. The connection with quantum criticality is established by switching on $t^{\prime}$. For positive $t^{\prime}$ a classical critical point emerges at finite temperature $T_{c}$, which increases with $t^{\prime}$. We note that $t^{\prime}$ is not the only control parameter that may be able to tune the critical point to finite temperatures, but other parameters, such as phonon coupling, may have the same effect. The phase diagram around the critical point is similar to that of the gas-liquid transition, where the incompressible Mott liquid and the compressible Mott gas are the coexisting phases. The strange metal region in this context may be viewed as the supercritical region lying in the vicinity of the critical point. Within the scenario we presented, the pseudogap region is not characterized by an order parameter, rather it must have the same symmetry as the Fermi-liquid and the marginal Fermi-liquid, since these regions are connected by an adiabatic path in the $T-\mu$ phase diagram. 
Further investigation is necessary to fully characterize the pseudogap region, and also to investigate the connection of those results with other scenarios of quantum criticality.

\section{Acknowledgements}

We would like to thank R. Gass, S. Kivelson, D. J. Scalapino, A. M. Tremblay, C. Varma, M. Vojt, S. R. White, J. Zaanen and for useful discussion that helped during the development of the presented work. This research was supported by NSF DMR-0706379, DOE CMSN DE-FG02-08ER46540, and by the DOE SciDAC grant DE-FC02-06ER25792. This research used resources of the National Center for Computational Sciences at Oak Ridge National Laboratory, which is supported by the Office of Science of the U.S. Department of Energy under Contract No. DE-AC05-00OR22725.

\section{References}

Anderson, P. W. 1987 The resonating valence bond state in $\mathrm{La}_{2} \mathrm{CuO} \mathrm{O}_{4}$ and superconductivity. Science, 235, 1196. (doi:10.1126/science.235.4793.1196)

Balakirev, F. F., Betts, J. B., Migliori, A., Tsukada, I., Ando, Y. \& Boebinger, G. S. 2009 Quantum Phase Transition in the Magnetic-Field-Induced Normal State of Optimum-Doped High- $T_{c}$ Cuprate Superconductors at Low Temperatures. Physical Review Letters, 102, 17 004. (doi:10.1103/PhysRevLett.102.017004)

Bardeen, J., Cooper, L. N. \& Schrieffer, J. R. 1957 Microscopic Theory of Superconductivity. Physical Review, 106, 162. (doi:10.1103/PhysRev.106.162)

Bednorz, J. \& Müller, K. 1986 Possible High $T_{c}$ Superconductivity in the Ba-LaCu-O System. Zeitschrift für Physik B, 64, 189-193. (doi:10.1007/BF01303701)

Bernhard, C., Tallon, J. L., Blasius, T., Golnik, A. \& Niedermayer, C. 2001 Anomalous Peak in the Superconducting Condensate Density of Cuprate High- $T_{c}$ Superconductors at a Unique Doping State. Physical Review Letters, 86, 1614. (doi:10.1103/PhysRevLett.86.1614)

Blankenbecler, R., Scalapino, D. J. \& Sugar, R. L. 1981 Monte Carlo calculations of coupled boson-fermion systems. I. Physical Review D, 24, 2278. (doi:10.1103/ PhysRevD.24.2278)

Broun, D. M. 2008 What lies beneath the dome? Nature Physics, 4, 170. (doi: $10.1038 /$ nphys909)

Chakraborty, S., Galanakis, D. \& Phillips, P. 2008 Emergence of Particle-Hole Symmetry near Optimal Doping in High-Temperature Copper Oxide Superconductors. arXiv:0807.2854. 6 pages: expanded version submitted to PRB.

Daou, R., Doiron-Leyraud, N., Leboeuf, D., Li, S. Y., Laliberté, F., Cyr-Choinière, O., Jo, Y. J., Balicas, L., Yan, J.-Q. et al. 2009 Linear temperature dependence of resistivity and change in the Fermi surface at the pseudogap critical point of a high- $T_{c}$ superconductor. Nature Physics, 5, 31. (doi:10.1038/nphys1109) 
Doiron-Leyraud, N., Proust, C., Leboeuf, D., Levallois, J., Bonnemaison, J.-B., Liang, R., Bonn, D. A., Hardy, W. N. \& Taillefer, L. 2007 Quantum oscillations and the Fermi surface in an underdoped high- $T_{c}$ superconductor. Nature, 447, 565. (doi:10.1038/nature05872)

Fauqué, B., Sidis, Y., Hinkov, V., Pailhès, S., Lin, C. T., Chaud, X. \& Bourges, P. 2006 Magnetic Order in the Pseudogap Phase of High- $T_{C}$ Superconductors. Physical Review Letters, 96, 197 001. (doi:10.1103/PhysRevLett.96.197001)

Frahm, H. \& Korepin, V. E. 1990 Critical exponents for the one-dimensional Hubbard model. Physical Review B, 42, 1070 399. (doi:10.1103/PhysRevB.42.10553)

Georges, A. \& Kotliar, G. 1992 Hubbard model in infinite dimensions. Physical Review B (Condensed Matter), 45, 6479. (doi:10.1103/PhysRevB.45.6479)

Georges, A., Kotliar, G., Krauth, W. \& Rozenberg, M. J. 1996 Dynamical mean-field theory of strongly correlated fermion systems and the limit of infinite dimensions. Reviews of Modern Physics, 68, 13. (doi:10.1103/RevModPhys.68.13)

Gubernatis, J. E., Jarrell, M., Silver, R. N. \& Sivia, D. S. 1991 Quantum Monte Carlo simulations and maximum entropy: Dynamics from imaginary-time data. Physical Review B, 44, 6011. (doi:10.1103/PhysRevB.44.6011)

Hettler, M. H., Mukherjee, M., Jarrell, M. \& Krishnamurthy, H. R. 2000 Dynamical cluster approximation: Nonlocal dynamics of correlated electron systems. Physical Review B, 61, 12 739. (doi:10.1103/PhysRevB.61.12739)

Hettler, M. H., Tahvildar-Zadeh, A. N., Jarrell, M., Pruschke, T. \& Krishnamurthy, H. R. 1998 Nonlocal dynamical correlations of strongly interacting electron systems. Physical Review B, 58, 7475. (doi:10.1103/PhysRevB.58.R7475)

Hinkov, V., Pailhès, S., Bourges, P., Sidis, Y., Ivanov, A., Kulakov, A., Lin, C. T., Chen, D. P., Bernhard, C. et al. 2004 Two-dimensional geometry of spin excitations in the high-transition-temperature superconductor $\mathrm{YB}_{2} \mathrm{Cu}_{3} \mathrm{O}_{6+x}$. Nature, 430, 650. (doi:10.1038/nature02774)

Hirsch, J. \& Fye, R. 1986 Monte Carlo method for magnetic impurities in metals. Physical review letters, 56, 2521. (doi:10.1103/PhysRevLett.56.2521)

Jarrell, M. 1992 Hubbard model in infinite dimensions: A quantum Monte Carlo study. Physical Review Letters, 69, 168. (doi:10.1103/PhysRevLett.69.168)

Jarrell, M. \& Gubernatis, J. E. 1996 Bayesian inference and the analytic continuation of imaginary-time quantum Monte Carlo data. Physics Reports, 269, 133. (doi:10.1016/0370-1573(95)00074-7)

Kawakami, N. \& Yang, S.-K. 1990 Correlation functions in the one-dimensional $t-J$ model. Physical Review Letters, 65, 2309. (doi:10.1103/PhysRevLett.65.2309)

Khatami, E., Mikelsons, K., Galanakis, D., Macridin, A., Moreno, J., Scalettar, R. T. \& Jarrell, M. 2010 Quantum criticality due to incipient phase separation in the two-dimensional Hubbard model. Physical Review B, 81(20), 201101. (doi:10.1103/PhysRevB.81.201101) 
Kotliar, G., Savrasov, S. Y., Pálsson, G. \& Biroli, G. 2001 Cellular Dynamical Mean Field Approach to Strongly Correlated Systems. Physical review letters, 87, 186 401. (doi:10.1103/PhysRevLett.87.186401)

Lieb, E. H. \& Wu, F. Y. 1968 Absence of Mott Transition in an Exact Solution of the Short-Range, One-Band Model in One Dimension. Physical Review Letters, 20, 1445. (doi:10.1103/PhysRevLett.20.1445)

Macridin, A. \& Jarrell, M. 2009 Isotope effect in the Hubbard model with local phonons. Physical Review B, 79(10), 104 517. (doi:10.1103/PhysRevB.79.104517)

Macridin, A., Jarrell, M. \& Maier, T. 2006 Phase separation in the Hubbard model using the dynamical cluster approximation. Physical Review B, 74(8), 085104. (doi:10.1103/PhysRevB.74.085104)

Maier, T., Jarrell, M., Pruschke, T. \& Hettler, M. 2005 Quantum cluster theories. Reviews of Modern Physics, 77, 1027. (doi:10.1103/RevModPhys.77.1027)

Metzner, W. \& Vollhardt, D. 1989 Correlated lattice fermions in $d=\infty$ dimensions. Physical review letters, 62, 324. (doi:10.1103/PhysRevLett.62.324)

Mikelsons, K., Khatami, E., Galanakis, D., Macridin, A., Moreno, J. \& Jarrell, M. 2009 Thermodynamics of the quantum critical point at finite doping in the twodimensional Hubbard model studied via the dynamical cluster approximation. Phys. Rev. B, 80(14), 140 505. (doi:10.1103/PhysRevB.80.140505)

Müller-Hartmann, E. 1989 a Correlated fermions on a lattice in high dimensions. $Z$. Phys. B (Condensed Matter), 74, 507-512.

Müller-Hartmann, E. $1989 b$ The Hubbard model at high dimensions: some exact results and weak coupling theory. Z. Phys. B (Condensed Matter), 76, 211-217.

Néel, L. 1949 Preuves expérimentales du ferromagnétisme et de l'antiferromagnétisme. Annales de l'institut Fourier, 1, 163-183.

Pathak, S., Chen, K.-S., Yang, S., Jarrell, M. \& Moreno, J. in preparation.

Phillips, P. 2010 Colloquium: Identifying the propagating charge modes in doped Mott insulators. Rev Mod Phys, 82(2), 1719-1742. (doi:10.1103/RevModPhys. 82.1719)

Platé, M., Mottershead, J. D., Elfimov, I. S., Peets, D. C., Liang, R., Bonn, D. A., Hardy, W. N., Chiuzbaian, S., Falub, M. et al. 2005 Fermi Surface and Quasiparticle Excitations of Overdoped $\mathrm{Tl}_{2} \mathrm{Ba}_{2} \mathrm{CuO} \mathrm{O}_{6+\delta}$. Physical Review Letters, 95, 077 001. (doi:10.1103/PhysRevLett.95.077001)

Radtke, R. J. \& Norman, M. R. 1994 Relation of extended Van Hove singularities to high-temperature superconductivity within strong-coupling theory. Phys. Rev. $B$, 50(13), 9554-9560. (doi:10.1103/PhysRevB.50.9554)

Rubtsov, A., Savkin, V. \& Lichtenstein, A. 2005 Continuous-time quantum Monte Carlo method for fermions. Phys. Rev. B, 72(3), 035 122. (doi:10.1103/ PhysRevB.72.035122) 
Sachdev, S. 2010 Where is the quantum critical point in the cuprate superconductors? physica status solidi (b), 247(3), 537-543. (doi:10.1002/pssb.200983037)

Shen, K. M., Ronning, F., Lu, D. H., Baumberger, F., Ingle, N. J. C., Lee, W. S., Meevasana, W., Kohsaka, Y., Azuma, M. et al. 2005 Nodal Quasiparticles and Antinodal Charge Ordering in $\mathrm{Ca}_{2-x} \mathrm{Na}_{x} \mathrm{CuO}_{2} \mathrm{Cl}_{2}$. Science, 307, 901. (doi: $10.1126 /$ science.1103627)

van der Marel, D., Molegraaf, H. J. A., Zaanen, J., Nussinov, Z., Carbone, F., Damascelli, A., Eisaki, H., Greven, M., Kes, P. H. et al. 2003 Quantum critical behaviour in a high- $T_{c}$ superconductor. Nature, 425, 271. (doi:10.1038/ nature01978)

Vidhyadhiraja, N. S., Macridin, A., Şen, C., Jarrell, M. \& Ma, M. 2009 Quantum Critical Point at Finite Doping in the 2D Hubbard Model: A Dynamical Cluster Quantum Monte Carlo Study. Physical Review Letters, 102, 206407. (doi:10. 1103/PhysRevLett.102.206407)

Xia, J., Schemm, E., Deutscher, G., Kivelson, S. A., Bonn, D. A., Hardy, W. N., Liang, R., Siemons, W., Koster, G. et al. 2008 Polar Kerr-Effect Measurements of the High-Temperature $\mathrm{YBa}_{2} \mathrm{Cu}_{3} \mathrm{O}_{6+x}$ Superconductor: Evidence for Broken Symmetry near the Pseudogap Temperature. Physical Review Letters, 100, 127 002. (doi:10.1103/PhysRevLett.100.127002)

Yang, S.-X., Fotso, H., Su, S.-Q., Galanakis, D., Khatami, E., She, J.-H., Moreno, J., Zaanen, J. \& Jarrell, M. 2010 Proximity of the Superconducting Dome and the Quantum Critical Point in the Two-Dimensional Hubbard Model. unpublished.

Zhang, F. C. \& Rice, T. M. 1988 Effective Hamiltonian for the superconducting $\mathrm{Cu}$ oxides. Physical Review B, 37, 3759. (doi:10.1103/PhysRevB.37.3759) 